\title{
FATORES PROGNÓSTICOS NAS GASTRECTOMIAS COM LINFADENECTOMIA D2 POR ADENOCARCINOMA GÁSTRICO
}

\author{
Prognostic factors in D2 gastrectomy for gastric adenocarcinoma \\ Osvaldo Antonio Prado CASTRO, Carlos Alberto MALHEIROS, \\ Francisco César Martins RODRIGUES, Elias Jirjoss ILIAS, Paulo KASSAB
}

ABCDDV/663

Castro OAP, Malheiros CA, Rodrigues FCM, Ilias EJ, Kassab P. Fatores prognósticos nas gastrectomias com linfadenectomia d2 por adenocarcinoma gástrico. ABCD Arq Bras Cir Dig 2009;22(3):158-64

RESUMO - Racional - A disseminação linfática é significativamente mais prevalente do que a hematogênica no câncer gástrico e assim torna-se importante o tratamento loco-regional, ou seja, a ressecção cirúrgica associada à linfadenectomia, preferencialmente do tipo D2. Objetivo - Avaliar a sobrevivência global nos pacientes submetidos à gastrectomia D2 por adenocarcinoma gástrico; determinar os principais fatores prognósticos e definir variáveis que possuam valor prognóstico independente. Métodos - Estudo prospectivo em 125 pacientes submetidos à gastrectomia D2, entre agosto de 1997 e outubro de 2005. A técnica adotada seguiu rigorosamente o protocolo proposto pelo Centro Nacional de Câncer de Tóquio. Resultados - Havia 73 homens e 52 mulheres com idades que variaram de 28 a 84 anos (média de 58,96 14,01). Setenta por cento das lesões situavam-se na porção distal do estômago, $20 \%$ eram proximais e $10 \%$ ocupavam os três segmentos anatômicos do órgão. Os estádios estavam assim distribuídos: I - 37 casos (29,6\%), II - 20 casos (16\%), III - 37 casos (29,6\%), e IV - 31 casos (24,8\%). Realizou-se 87 gastrectomias subtotais e 38 totais. A morbidade total foi de $26,4 \%$, constituindo-se predominantemente de fístulas e complicações pulmonares. A letalidade foi de 9,6\%. Após seguimento médio de 48 meses, 68 (54,4\%) pacientes tinham falecido, representando sobrevivência global de 45,6\%. As análises univariada e multivariada revelaram que: tumores que acometiam grandes segmentos do estômago, lesões que acometiam além da serosa (T3 ou T4), comprometimento neoplásico em mais de sete linfonodos (N2 ou N3), presença de metástases à distância (M1) e o estádio III e IV da doença, estavam relacionados diretamente com pior prognóstico. Conclusão - Menos da metade dos pacientes encontrava-se vivo após seguimento médio de quatro anos; o estádio TNM isolado constituiu-se no principal fator prognóstico, sendo que a extensão do comprometimento tumoral na câmara gástrica, o estádio da doença, a ocorrência de metástases a distância e principalmente a presença de metástases linfonodais, constituíram-se em variáveis com valor prognóstico independente.

DESCRITORES - Neoplasias gástricas. Adenocarcinoma. Gastrectomia. Excisão de linfonodo. Prognóstico.

\section{INTRODUÇÃO}

O único tratamento curativo eficiente para o câncer do estômago é a ressecção que pode ser endoscópica em alguns tumores limitados à mucosa, raros em nosso meio, mas que, via de regra, é cirúrgica na maior parte ${ }^{9,29}$. Também como tratamento paliativo, parece que a ressecção seja, ainda, um dos melhores tratamentos ${ }^{21}$, talvez não pelo aumento da sobrevivência média em longo prazo, mas certamente, no aprimoramento da qualidade de vida ${ }^{5}$.

Uma vez que a disseminação linfática é significativamente mais prevalente do que a hematogênica ${ }^{2}$, torna-se inquestionável que o tratamento loco-regional, ou seja, a ressecção cirúrgica associada à linfadenectomia, preferencialmente do tipo D2, permanece sendo o melhor e mais eficiente tratamento entre todos os disponíveis ${ }^{23,32}$.

Entretanto, muitas questões são colocadas com relação à linfadenectomia D2. A mais frequentemente citada nos últimos anos tem sido a morbidade e letalidade

Trabalho realizado Disciplina de Cirurgia Geral da Faculdade de Medicina da Universidade de Santo Amaro, SP, Brasil

Endereço para correspondência: Osvaldo Antonio Prado Castro, e-mail: oapcastro@gmail.com relacionadas a esse procedimento. $\mathrm{O}$ assunto ganhou evidência após as publicações dos estudos Britânico ${ }^{8}$ e Holandês ${ }^{3}$ que demonstraram em análises prospectivas e randomizadas, que a linfadenectomia D2 aumentava significativamente as complicações e a mortalidade operatória, anulando, assim, os possíveis benefícios da ressecção linfática mais extensa.

O desmembramento mais criterioso destas publicações revelou que além do baixo volume cirúrgico anual de alguns centros participantes, a esplenectomia e a pancreatectomia foram os fatores responsáveis pelos maus resultados, sendo que, esses dois procedimentos aparentemente foram realizados desnecessariamente em muitos pacientes daquelas séries ${ }^{18}$.

$\mathrm{O}$ aumento na sobrevida tem sido demonstrado por vários serviços em todo o mundo quando se realiza a linfadenectomia D2 ${ }^{24}$. A sobrevivência global em cinco anos no Japão, utilizando apenas o tratamento cirúrgico, chegou a $71 \%$, enquanto que na Itália, foi de $42 \%$ mesmo com a utilização de terapêuticas adjuvantes como a radioterapia e a quimioterapia ${ }^{29}$. Mesmo em tumores sem comprometimento linfonodal, ou seja, N0, a realização da linfadenectomia D2 apresentou índices de sobrevivência em 5 e 10 anos significativamente melhores do que com 
a linfadenectomia D1, 97 e $95 \%$ ante 90 e $81 \%$ respectivamente.

Se por um lado os resultados referentes à sobrevivência com a linfadenectomia D2 são conflitantes, é inquestionável a sua importância para a adequada determinação do estádio. Erros na correta determinação do estádio, frequentemente associados às linfadenectomias insuficientes, podem resultar em migrações de estádio, influenciando negativamente a análise dos resultados cirúrgicos e da sobrevivência ${ }^{35}$. A significância disso tem sido claramente demonstrada em quase todas as séries que definem o estádio da doença como sendo a principal variável prognóstica.

Observa-se que o ponto de convergência da maioria das publicações atuais sobre o câncer do estômago, reside na identificação e na verificação de significância estatística dos principais fatores prognósticos, fundamentais para a escolha da melhor abordagem terapêutica ${ }^{26}$. A consideração desses fatores de risco deve ser muito criteriosa, uma vez que, as características epidemiológicas peculiares de uma determinada população podem não se aplicar à outra.

Apesar de encontrarem-se artigos em profusão na literatura, a quantidade de análises desse tipo em nosso país, é relativamente restrita, o que motivou a empreender esta análise.

Os objetivos do presente estudo, nos pacientes submetidos à gastrectomia com linfadenectomia D2 por adenocarcinoma gástrico, foram: 1) avaliar a sobrevivência global nos pacientes submetidos à gastrectomia com linfadenectomia D2; 2) determinar os principais fatores prognósticos; 3) definir variáveis que possuam valor prognóstico independente.

\section{MÉTODOS}

De agosto de 1997 a outubro de 2005, 125 pacientes com diagnóstico de adenocarcinoma gástrico foram submetidos à gastrectomia com linfadenectomia D2 na Disciplina de Cirurgia Geral da Faculdade de Medicina da Universidade de Santo Amaro, SP, Brasil.

Os dados dos pacientes foram cuidadosamente registrados em um protocolo adaptado do Centro Nacional de Câncer de Tóquio. Todos os pacientes foram operados por quatro cirurgiões que seguiram rigorosamente a metodologia proposta pela Associação Japonesa de Câncer Gástrico.

A operação consistia na determinação exata do local da lesão na câmara gástrica; avaliação da extirpabilidade do tumor; determinação dos grupos linfonodais a serem ressecados para a correta execução da dissecção D2; realização da ressecção gástrica que poderia ser total ou subtotal dependendo da localização do tumor; reconstrução que nas gastrectomias subtotais poderia ser do tipo Billroth I ou II ou Kenneth-Warren, e nas totais, Y de Roux, Kenneth-Warren ou Safatle.

Durante a operação os grupos linfonodais e outros tecidos que, eventualmente fossem ressecados, eram separados em frascos individuais para envio à patologia. As peças ressecadas eram rigorosamente dissecadas e estudadas ao final da operação pelo próprio cirurgião, com posterior preenchimento meticuloso do protocolo.

Adotou-se primariamente o sistema de estadiamento proposto pela Associação Japonesa de Câncer Gástrico ${ }^{14}$, entretanto, para a realização da análise estatística, empregou-se o estádio proposto pela União Internacional Contra o Câncer ${ }^{33}$.

Todos os pacientes foram seguidos individualmente no Ambulatório de Cirurgia do Estômago e Duodeno da Faculdade de Medicina de Santo Amaro. No primeiro ano trimestralmente e a partir do segundo ano, de modo semestral.

Os seguintes parâmetros foram analisados: idade, gênero, local da lesão, extensão da ressecção, profundidade da lesão $(\mathrm{T})$, acometimento linfonodal $(\mathrm{N})$, presença de metástases $(\mathrm{M})$, estádio, total de linfonodos extirpados, tipo de reconstrução, ressecções combinadas, perda sanguínea e necessidade de hemotransfusões, tempo cirúrgico, morbidade, letalidade, tempo de sobrevivência e presença de câncer na época do óbito.

Respeitando a nomenclatura preconizada pela Associação Japonesa de Câncer Gástrico ${ }^{14}(\mathrm{~L}$ - terço distal; $\mathrm{M}$ - terço médio; $\mathrm{U}$ - terço superior; D - duodeno; e E - esôfago), as lesões foram agrupadas, de acordo com a sua localização predominante na câmara gástrica, em três grupos distintos: Grupo 1 (lesões distais) - L, LD, LM ou ML; Grupo 2 (lesões extensas que compreendem os 3 segmentos do estômago) - LMU, UML, MLU ou MUL; Grupo 3 (lesões proximais) - UM, MU, M, U ou EU.

Da mesma forma, com relação ao estádio, suprimiramse as subdivisões do estádio I (IA e IB) e do estádio III (IIIA e IIIB), permanecendo quatro estádios distintos: I, II, III e IV.

Para a análise estatística foram empregados os testes de qui-quadrado e Exato de Fisher, curva de sobrevivência de Kaplan-Meier, modelos de regressão de Cox uni e multivariados. O nível de significância foi de $5 \%$. O pacote estatístico utilizado foi o SPSS 14.0 for Windows.

\section{RESULTADOS}

Houve discreto predomínio do gênero masculino, responsável por $58,4 \%$ da casuística. A idade média dos pacientes foi de 58,96 anos (28 a 84 anos, dp - $\pm 14,01$ ), sendo que $41,6 \%$ da população estudada tinham acima de 65 anos.

Quanto à localização das lesões de acordo com os grupos definidos na metodologia deste estudo, observou-se que em 88 casos $(70,4 \%)$ os tumores eram distais (Grupo 1), 25 casos (20\%) lesões proximais (Grupo 3), e em 12 pacientes $(9,6 \%)$ neoplasias que comprometiam os três segmentos do órgão (Grupo 2).

A ressecção do tipo subtotal foi realizada $69,6 \%$ dos pacientes, sendo que a estratificação por estádios foi: estádio I - 29,6\%, II - 16\%, III - 29,6\% e IV - 24,8\%.

A taxa de morbidade foi de $26,4 \%$, constatados em 28 pacientes, sendo que em cinco casos tiveram mais de uma complicação. As fístulas (13\%) e as afecções pulmonares (6\%), pneumonias ou síndrome da angústia respiratória 
aguda, estiveram entre as mais frequentes.

Foram realizadas ressecções combinadas à gastrectomia D2 em 53 pacientes. O baço (27), a vesícula biliar (18) e o segmento corpo-caudal do pâncreas (9) foram os órgãos mais frequentemente extirpados.

Extirparam-se 3874 linfonodos, perfazendo uma média de 31 linfonodos por caso, sendo que, 794 estavam comprometidos ( $\mathrm{N} 0-40,8 \%, \mathrm{~N} 1-24 \%, \mathrm{~N} 2-20 \%, \mathrm{~N} 3-15,2 \%$ ).

O tempo cirúrgico médio das operações foi de 222,44 minutos, sendo de 227,07 minutos nas gastrectomias subtotais e 211,84 minutos nas gastrectomias totais.

A mortalidade operatória foi de $9,6 \%$. A sobrevivência global, independente do estádio, foi de $45,6 \%$, sendo que na época do óbito, dos 56 pacientes que morreram 43 tinham a presença da doença.

Nos três grupos definidos, não houve diferença estatística na idade média dos pacientes, assim como, nos estádios em que as doenças se encontravam, nem na ocorrência de metástases à distância. Já, a ocorrência de metástases linfonodais em mais de 15 linfonodos (N3), foi significativamente maior nos tumores que comprometiam grandes porções do estômago (Grupo 2). A letalidade foi significativamente menor nas neoplasias distais (Grupo 1). A sobrevivência foi nula no grupo 2, além de estarem relacionados com maiores índices de recidiva tumoral à época da morte (Tabela 1).

TABELA 1 - Correlação dos locais com letalidade, sobrevivência, presença de câncer à época do óbito e ocorrência de metástases linfonodais em 125 pacientes submetidos à gastrectomia D2. FCMSCSP, 2008

\begin{tabular}{|c|c|c|c|}
\hline Variáveis & $\begin{array}{c}\text { Grupo } 1 \\
N^{\circ}(\%)\end{array}$ & $\begin{array}{c}\text { Grupo } 2 \\
N^{\circ}(\%)\end{array}$ & $\begin{array}{c}\text { Grupo } 3 \\
N^{\circ}(\%)\end{array}$ \\
\hline \multicolumn{4}{|l|}{ Letalidade } \\
\hline Sim & $3(3,4)$ & $2(16,7)$ & $7(28,0)$ \\
\hline Não & $85(96,6)$ & $10(83,3)$ & $18(72,0)$ \\
\hline \multicolumn{4}{|c|}{ Sobrevivência ( \pm 48 meses) } \\
\hline Morto & $39(44,3)$ & $12(100)$ & $68(54,4)$ \\
\hline Vivo & $49(55,7)$ & $0(0)$ & $57(45,6)$ \\
\hline \multicolumn{4}{|c|}{ Presença de câncer na época do óbito } \\
\hline Sim & $27(30,7)$ & $9(75,0)$ & $7(28,0)$ \\
\hline Não & $58(65,9)$ & $1(8,3)$ & $11(44,0)$ \\
\hline Indefinido & $3(3,4)$ & $2(16,7)$ & $7(28,0)$ \\
\hline \multicolumn{4}{|c|}{ Metástases linfonodais (N) } \\
\hline $\mathrm{N}_{0}$ & $41(46,6)$ & $2(16,7)$ & $8(32,0)$ \\
\hline $\mathrm{N}_{1}$ & $18(20,5)$ & $1(8,3)$ & $11(44,0)$ \\
\hline $\mathrm{N}_{2}$ & $19(21,6)$ & $3(25,0)$ & $3(12,0)$ \\
\hline $\mathrm{N}_{3}$ & $10(11,4)$ & $6(50,0)$ & $3(12,0)$ \\
\hline
\end{tabular}

$\mathrm{P}<0,001$ em letalidade e sobrevivência

$\mathrm{P}<0,05$ em presença de câncer na época do óbito e metástases linfonodais

Constatou-se que a maioria das mortes relacionadas ao câncer ocorreu em pacientes que eram portadores de tumores que invadiam além da camada serosa (T3 ou T4), comprometiam mais do que sete linfonodos (N2 ou N3), e eram pertencentes aos estádio III ou IV (Tabela 2).

Da mesma forma, quanto à sobrevivência mediana, verificou-se que foi nitidamente superior nos tumores que se localizavam na porção distal do estômago (Grupo 1); estavam limitados à camada mucosa, submucosa ou até mesmo muscular própria ( $\mathrm{T} 1$ e $\mathrm{T} 2$ ); apresentavam no
TABELA 2 - Ocorrência de óbitos em relação à profundidade dos tumores, acometimento linfonodal e por estádio (excluídas as mortes nos primeiros 30 dias) em 125 pacientes submetidos à gastrectomia D2. FCMSCSP, 2008

\begin{tabular}{cccc}
\hline Variáveis & No de casos & No de óbitos & $\begin{array}{c}\text { Pacientes vivos } \\
\text { N }(\%)\end{array}$ \\
\hline T & & & \\
1 & 22 & $1(4,5 \%)$ & $21(95,5 \%)$ \\
2 & 27 & $6(22,3 \%)$ & $21(77,8 \%)$ \\
3 & 55 & $31(56,4 \%)$ & $24(43,6 \%)$ \\
4 & 9 & $5(55,6 \%)$ & $4(44,4 \%)$ \\
\hline N & & & \\
$N_{0}$ & 49 & $8(16,3 \%)$ & $41(83,7 \%)$ \\
$\mathrm{N}_{1}$ & 25 & $8(32,0 \%)$ & $17(68,0 \%)$ \\
$\mathrm{N}_{2}$ & 24 & $16(66,7 \%)$ & $8(33,3 \%)$ \\
$\mathrm{N}_{3}$ & 15 & $11(73,3 \%)$ & $4(26,7 \%)$ \\
\hline Estádio & & & \\
I & 36 & $3(8,3 \%)$ & $33(91,7 \%)$ \\
II & 19 & $5(26,3 \%)$ & $14(73,7 \%)$ \\
III & 32 & $18(56,2 \%)$ & $14(43,8 \%)$ \\
IV & 26 & $17(65,4 \%)$ & $9(34,6 \%)$ \\
\hline Total & 113 & 43 & $70(61,9 \%)$ \\
\hline
\end{tabular}

$\mathrm{P}<0,05$

máximo seis linfonodos comprometidos ( $\mathrm{N} 0$ e N1); e eram pertencentes ao estádio I ou II (Figura 1).
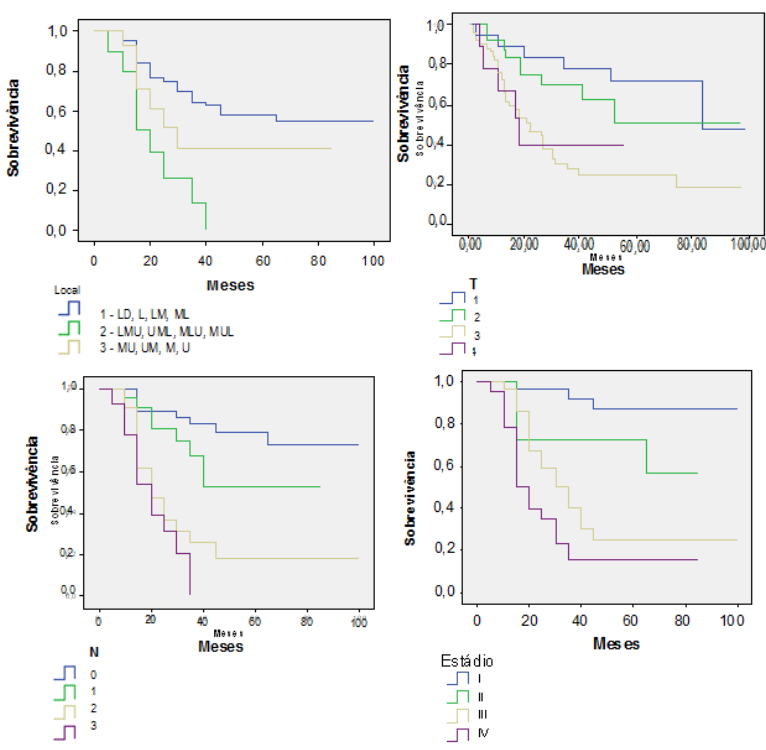

FIGURA 1 - Curva de sobrevivência de acordo com a localização das lesões, profundidade tumoral (T), comprometimento linfonodal $(\mathrm{N})$ e estádio em 125 pacientes submetidos à gastrectomia D2. FCMSCSP, 2008

A análise univariada revelou significância estatística para: lesões que comprometem grandes porções do estômago (Grupo 2), tumores com metástases em mais de 7 linfonodos regionais (N2 e N3), presença de metástases à distância (M1), e neoplasias em estádio III e IV; já, em relação as perdas sanguíneas intra-operatórias que variaram entre 100 e $1200 \mathrm{ml}$, apesar de uma forte tendência não revelou significância estatística; quanto a necessidade de 
hemotransfusão, não se verificou essa significância.

A análise multivariada constatou que os tumores que comprometem os três segmentos do estômago, os estádios III e IV da doença e principalmente o comprometimento linfonodal constituem-se em variáveis com significância estatística independente.

\section{DISCUSSÃO}

Dentre os fatores prognósticos muitos são relacionados à doença e ao doente, como por exemplo, tipo histológico, agressividade do tumor, localização, padrão de disseminação, estádio à época do diagnóstico, idade e presença de doenças associadas entre outras ${ }^{26}$. Outros fatores estão relacionados ao tratamento oferecido ao paciente, em resumo à cirurgia em si que constitui o principal método com intenção curativa nos cânceres precoces e o único método curativo nos cânceres avançados.

A nosso ver, muitos grupos relacionam a radicalidade da cirurgia somente à ressecção, entretanto, é imperativo dizer que a radicalidade está muito mais relacionada à linfadenectomia do que à extensão da ressecção.

O planejamento da linfadenectomia deve ser cuidadosamente traçado no pré-operatório e no intra-operatório, baseado no local do tumor, no estádio e na curabilidade do procedimento. De fato, o local da lesão no estômago, além de indicar as cadeias linfáticas a serem retiradas, também está relacionado com os resultados cirúrgicos e a sobrevivência. $\mathrm{O}$ acometimento de todo o estômago leva a maiores taxas de mortalidade operatória quando comparado com tumores restritos a um segmento gástrico; além de apresentar menores taxas de sobrevivência em cinco e dez anos. Da mesma forma, os cânceres proximais tendem a apresentar maiores taxas de morbi-mortalidade, tais como fístulas e afecções pulmonares, que se atribui à própria gastrectomia total e a ressecção de outros órgãos ${ }^{22}$.

A determinação do estádio talvez seja o fator mais importante no planejamento cirúrgico, entretanto parece ser mais importante ainda o seu papel na caracterização do prognóstico, como se constatou na análise multivariada realizada que revelou diferença significativa na sobrevivência nos diferentes estádios ${ }^{4}$.

A migração de estádio conhecida como fenômeno de Will Rogers ocorre quando os pacientes mudam de estádio devido a variações do acometimento linfonodal ${ }^{4}$. Isso foi observado em nosso meio quando se simulou linfadenectomia D1 em pacientes submetidos à dissecção D2. Quando se comparam linfadenectomias mais amplas à D3 com D1, houve uma migração de $8 \%$ dos pacientes N2 para N3. Uma análise similar revelou uma migração relacionada ao $\mathrm{N}$ de $20,7 \%{ }^{19}$.

Acreditamos, portanto, que a maior causa de erro no estadiamento relativamente ao $\mathrm{N}$ deve-se a linfadenectomias econômicas, o que não ocorreu no presente estudo, haja vista que, uma média de 31 linfonodos foram ressecados por caso, sendo que a literatura recomenda que o número mínimo de linfonodos a serem analisados quando se utiliza o sistema da UICC seja de $20^{13}$. A importância da linfadenectomia é tanta, que a avaliação quantitativa do comprometimento linfonodal no nível 2 é considerado como sendo um dos fatores prognósticos mais poderosos. A sobrevida em 5 anos de pacientes que tem entre 0 e 1 linfonodo comprometido no nível 2 é de $63,1 \%$ e $57,1 \%$ respectivamente, ao passo que, quando hão metástases em 2 ou mais linfonodos neste nível, a sobrevivência em 5 anos decresce drasticamente para aproximadamente $17 \%{ }^{1}$.

Na presente análise, o acometimento linfonodal seja no nível 2 ou 3, mostrou ser um importante fator prognóstico independente.

Acredita-se que haja uma correlação importante entre o tamanho das lesões e a extensão do comprometimento linfonodal. Kikuchi et al. ${ }^{16}$, demonstraram que tumores maiores do que $6 \mathrm{~cm}$ estavam relacionados a uma maior incidência de comprometimento linfático no nível 2 e 3 . De fato, na casuística estudada, constatou-se que neoplasias que acometiam os três segmentos do estômago apresentaram significativamente maior comprometimento linfonodal no nível 2 (25\%) e 3 (50\%). Assim sendo, como em nosso meio a incidência de tumores avançados e extensos é relativamente alta, a necessidade de dissecção de grupos linfonodais mais distantes torna-se obrigatória.

As evidências têm apontado que a gastrectomia D2 beneficia globalmente os pacientes com adenocarcinoma gástrico, entretanto parece que nos pacientes pertencentes ao estádio III essa melhoria na sobrevivência em longo prazo seja ainda mais consistente, de 14 meses nas dissecções D1 para 33,8 meses nas D2. Isso é extremamente relevante em nosso meio onde identificamos claramente uma elevada incidência de neoplasias em estádio III, o que na presente casuística, correspondeu a 30\% dos pacientes.

Um aspecto interessante que observamos em nosso estudo, foi que a recidiva jamais se deu nos linfonodos, sempre ocorreu no peritônio ou à distância. As lesões esquirrosas apresentaram uma incidência significativamente maior de recidiva local, sendo que, o fator de maior impacto para essa ocorrência, foi o estádio da doença. Já para outros autores, os fatores envolvidos na recidiva tardia estão relacionados ao potencial de cura, à profundidade da lesão e muito provavelmente a fatores relacionados à resistência do hospedeiro ${ }^{55}$. Estima-se que $80 \%$ das recidivas ocorrem nos 2 primeiros anos da operação, sendo que, após diagnóstico da recidiva peritoneal a sobrevida média é de apenas 4,9 meses. Assim, uma adequada cirurgia do ponto de vista linfático, contribui decisivamente no controle locorregional da doença, praticamente eliminando esse fator como sendo uma causa de recidiva ${ }^{28}$.

Desde a década de 60, os países Ocidentais apresentam morbidade e mortalidade cirúrgicas mais elevadas do que o Oriente. Em publicação alemã, Rohde et al. ${ }^{27}$, avaliaram as causas de mortalidade intra-hospitalar em 1423 pacientes foram submetidos a algum procedimento cirúrgico, paliativo ou curativo. Constataram que as complicações consideradas cirúrgicas, tais como fístulas, abscessos e infecção de sítio cirúrgico, foram responsáveis por 5,6\% dos óbitos; já as complicações clínicas, entre elas cardíacas, pulmonares e renais, foram responsáveis por $51,8 \%$ das mortes. Os au- 
tores correlacionaram esses índices de morbi-mortalidade com a extensão e a localização das lesões no estômago e notaram que, pacientes com tumores que acometiam todo o órgão apresentaram mortalidade de $13,6 \%$ contra 7,8\% nos tumores distais sendo que, as gastrectomias totais acarretaram a uma mortalidade de 10,4\%. Esses resultados são semelhantes aos da presente casuística que constatou uma taxa de letalidade de $16,7 \%$ nos pacientes com lesões que compreendiam os três segmentos do órgão, ante 3,4\% nas lesões distais. Já nas lesões de localização proximal, a letalidade foi de $28 \%$, sendo que, um dos fatores que podem ser atribuídos a este elevado valor é a correlação com a própria realização da gastrectomia total. De fato, quando se analisaram os pacientes submetidos à ressecção total do estômago a taxa de mortalidade foi de $21,05 \%$ contra $4,59 \%$ nas gastrectomias subtotais.

As discussões envolvendo a morbidade e a mortalidade cirúrgicas relacionadas à linfadenectomia D2, ainda estão em evidência. Duas publicações motivaram essa celeuma, os estudos Holandeses e Britânicos. Ambos prospectivos e multicêntricos, sendo que o primeiro teve $10 \%$ de mortalidade na D2 contra 4\% na D1 $(P=0,004)$ e morbidade de $43 \%$ na D 2 contra $25 \%$ na D1 $(P<0,001)$. Essas taxas, assim que publicadas, chamaram a atenção da comunidade científica já que praticamente anulavam os benefícios da linfadenectomia na sobrevivência em longo prazo. O desmembramento desses estudos cocluiu que dois fatores haviam influenciado esses resultados, a realização de esplenectomia e pancreatectomias em alta freqüência e o baixo número de cirurgias realizadas por significativa parcela dos centros participantes ${ }^{11}$.

Não há dúvidas de que a indicação de cirurgias alargadas com ressecções multiorgânicas deve ser bastante criteriosa. Existem evidências consistentes na literatura que as ressecções combinadas, principalmente a esplenectomia e a pancreatectomia, estejam intimamente relacionadas com maiores índices de morbi-mortalidade. Griffith et al. ${ }^{10}$, observou que a mortalidade quando se realizou a esplenectomia, chegou a $12 \%$ sendo que permaneceu em $2,5 \%$ quando não; o mesmo ocorreu em relação às complicações pós-operatórias, $41 \%$ contra $14 \%$. Mais recentemente, Lo et al. ${ }^{22}$ constataram taxas de morbidade de $11,9 \%$ nas gastrectomias sem ressecções combinadas ante $26,8 \%$ nos pacientes submetidos à essas ressecções. Nesta casuística, observamos essa mesma tendência, a morbidade nos pacientes que não foram submetidos a ressecções combinadas foi de $15,27 \%$, já nos que foram submetidos, esse índice chegou a $32,07 \%$. Outros autores identificaram diferenças ainda mais significativas, Yamamoto et al ${ }^{36}$, notaram morbidade de $1,7 \%$ nas gastrectomias isoladas e de $17,5 \%$ nas combinadas; já para Weitz et al..$^{34}$, esses números chegaram a $0 \%$ e $59 \%$, respectivamente.

É bastante comum, a realização de pancreatectomia e esplenectomia associada à gastrectomia total, particularmente nos tumores T3 ou T4 com o objetivo de se extrair os linfonodos do grupo 10 e $11 \mathrm{~d}$. Entretanto, é importante relatar que a ressecção do baço e do pâncreas para a retirada desses linfonodos, não e um pré-requisito, levando a maio- res índices de fístula pancreática, abscessos sub-frênicos e complicações pulmonares. Geralmente a indicação da ressecção desses órgãos restringe-se aos casos em que se constate uma invasão direta pela neoplasia. Ocorre, no entanto, que as metástases linfáticas para o grupo 10 só ocorrem em tumores avançados na porção superior da grande curvatura e a dissecção do grupo 11d é perfeitamente factível preservando-se o pâncreas ${ }^{2}$. Já a preservação do baço para a dissecção do grupo 10, entretanto, é bastante difícil sendo apenas realizada por pequena parcela de cirurgiões no mundo e, segundo nosso conhecimento, por nenhum grupo no Brasil ${ }^{20}$.

Atualmente, estão em evidência estudos clínicos que visam determinar um índice prognóstico baseado não somente em dados obtidos a partir de espécimes cirúrgicos ou relacionados ao procedimento cirúrgico em si, mas sim propõe valorizar aspectos clínicos, individuais de cada paciente ${ }^{25}$.

Fatores como a idade do paciente, presença de vícios como o tabagismo e o alcoolismo, além do tipo histológico e da localização da lesão, tinham igual valor estatístico do que o consagrado estadiamento TNM.

Uma publicação nacional, com 230 pacientes, identificou 6 variáveis com valor significativo e independente na sobrevivência. O sexo do paciente, a presença de perda ponderal, a contagem de linfócitos, o estádio TNM, a realização da linfadenectomia e a razão de linfonodos comprometidos, foram incluídos como variáveis em um escore prognóstico com eficiência significativamente superior, do que em relação ao estadiamento TNM isolado ${ }^{6}$.

Schwarz RE, et al. ${ }^{30}$, analisando as diferenças nas taxas de sobrevivência em populações etnicamente diferentes, concluíram que os fatores responsáveis para esse aspecto estavam relacionados principalmente ao local da lesão, extensão da ressecção gástrica e a presença de comorbidades. Já Shiraishi et al. ${ }^{31}$, analisando tumores extensos, maiores do que $10 \mathrm{~cm}$, e correlacionando-os com 24 variáveis clinicopatológicas, relacionadas aos achados referentes aos pacientes, ao tratamento cirúrgico e a análise histopatológica, concluíram que, nenhum fator clínico nem cirúrgico, até mesmo a extensão da linfadenectomia, pôde ser associado, do ponto de vista estatístico, a sobrevida em 5 anos. Mais uma vez, somente os dados anátomopatológicos, referentes ao TNM, tiveram forte relação com a sobrevivência dos pacientes.

Com uma casuística de 697 pacientes, Yokota et al. ${ }^{37}$, observaram que o tamanho do tumor, é um fator clínico preditivo de sobrevivência, entretanto, a análise multivariada o excluiu como variável de valor prognóstico independente. Nossa casuística não analisou especificamente o tamanho das lesões tumorais, entretanto demonstrou que as lesões que comprometem grandes porções do estômago estão significativamente relacionadas com um mau prognóstico.

Apesar dos avanços expressivos na área da cirurgia gástrica oncológica, tanto no que diz respeito à ressecção, dissecção linfonodal e reconstrução, chegando ao ponto de muitos pesquisadores afirmarem que tenha atingido o 
nível máximo de evolução, o mesmo não ocorre quando nos referimos aos tratamentos complementares, sejam neoadjuvantes ou adjuvantes. No momento percebe-se uma nítida tendência mundial em não se cogitar tratar o câncer do estômago somente com a ressecção cirúrgica, mas sim, associada a alguma modalidade adjuvante seja quimio ou radioterápica, entretanto, os resultados ainda são extremamente contraditórios de modo que, outras variáveis, devam estar influenciando estes resultados, daí a importância em se determinar adequadamente os fatores prognósticos ${ }^{7}$. Assim sendo, expressões como terapia individualizada ou sob medida tem sido cada vez mais mencionadas ${ }^{17}$.

O futuro do tratamento do paciente com câncer do estômago certamente necessitará de uma abordagem multidisciplinar mais ampla, porém com foco cada vez mais individualizado de cada doente. Embora a cirurgia curativa seja a opção terapêutica mais desejada, quando se trata de um paciente portador de um câncer gástrico avançado, a sua evolução nem sempre, ou melhor, raramente revela ser curativa.

\section{CONCLUSÕES}

Os resultados obtidos nas condições de realização do presente trabalho, permitiram as seguintes conclusões:

- Considerando-se os quatro estádios da doença e um seguimento médio de 4 anos, pouco menos da metade dos pacientes encontravam-se vivos.

- O estádio TNM isolado constituiu-se no principal fator prognóstico

- As variáveis que possuíram valor prognóstico independente foram:

o Comprometimento linfonodal

o Extensão do comprometimento tumoral no estômago

o Estádio avançado da doença

Castro OAP, Malheiros CA, Rodrigues FCM, Ilias EJ, Kassab P. Prognostic factors in D2 gastrectomy for gastric adenocarcinoma. ABCD Arq Bras Cir Dig 2009;22(3):158-64

ABSTRACT - Background - Lymphatic spread is more common in gastric cancer than the hematogenic one. Thus, the locoregional dissection type D2 seems to be important. Aim - To evaluate the overall survival after D2 gastrectomy for gastric adenocarcinoma and to determine the most important prognostic factors, including those with independent statistical value. Methods - Prospective study with 125 patients operated between August 1997 and October 2005. The technique employed followed strictly the protocol of the National Cancer Center - Tokyo. Results - There were 73 men and 52 women with ages ranging 28 to 84 years (mean of $58.96 \pm 14.01$ ). Seventy per cent of the lesions were located at the distal portion of the stomach, $20 \%$ were proximal and $10 \%$ comprised the whole organ. The stage distributions were: I - 37 cases (29.6\%), II - 20 cases (16\%), III -37 cases $(29.6 \%)$, and IV -31 cases $(24.8 \%)$. It was performed the amount of 73 subtotal gastrectomies and 52 totals. The morbidity rate was $26.4 \%$, mainly, due to leakage and pulmonary complications. The overall mortality rate was $9.6 \%$. After a mean follow-up of 48 months, 68 (54.4\%) patients were dead, representing an overall survival rate of $45.6 \%$. Univariate and multivariate statistical analysis revealed that: tumors comprising the whole stomach, beyond the serosal layer (T3 or T4), with more than seven metastatic lymph nodes (N2 or N3), with distant metastasis (M1), and belonging to the stage III or IV of the disease, were related to a poor prognosis. Conclusions - Less than a half of the patients were alive after a mean follow-up of almost four years; the TNM stage system was the main prognostic factor, ergo, the extension of the lesions, the stage of the disease, metastatic occurrence and mainly the lymph node involvement were define as an independent prognostic factors.

HEADINGS - Stomach neoplasm. Adenocarcinoma. Gastrectomy. Lymph node excision. Prognosis.

\section{REFERÊNCIAS}

1. Adachi Y, Shiraishi N, Suematsu T, Shiromizi A, Yamaguchi K, Kitano S. Most important lymph node information in gastric cancer: multivariate prognostic study. Ann Surg Oncol. 2000;7:503-7.

2. Bjelovic MM Pesko P, Sabljak P, Stojakov D, Simic A, Velickovic D, et al. Dissection of the lymph nodes in the splenic hilus and along splenic artery. In: Abstracts of 6th International Gastric Cancer Congress, Yokohama, Japan, May 4-7, 2005. p.107.

3. Bonenkamp JJ, Songun I, Hermans J, Sasako M, Welvaart K, Plukker JT, et al. Randomised comparison of morbidity after D1 and D2 dissection for gastric cancer in 996 Dutch patients. Lancet. 1995;345:745-8.

4. Bunt AM, Hermans J, Smit VT, van de Velde CJ, Fleuren GJ, Bruijn JA. Surgical/pathologic-stage migration confounds comparisons of gastric cancer survival rates between Japan and Western countries. J Clin Oncol. 1995;13:1925.

5. Castro OAP, Ilias EJ, Kassab P, Lee AD, Ketzer B, Pompeo A, et al. No benefit in palliative surgery for survival of unresectable gastric cancer [OR-12-151A]. In: Gama-Rodrigues J, Kassab P. Proceedings of the Seventh International Gastric Cancer Congress; 2007 May 9-12; São Paulo: International Gastric Cancer Association; 2007. p.82.

6. Costa ML, Ribeiro KCB, Machado MA, Costa AC, Montagnini AL. Prognostic score in gastric cancer: the importance of a conjoint analysis of clinical, pathologic, and therapeutic factors. Ann Surg Oncol. 2007;13:843-50.

7. Cunningham D, Allum WH, Stenning SP, Thompson JN, Van de Velde CJ, Nicolson $\mathrm{M}$, et al. Perioperative chemotherapy versus surgery alone for resectable gastroesophageal cancer. N Engl J Med. 2006;355(1):11-20.
8. Cuschieri A, Fayers P, Fielding J, Craven J, Bancewicz J, Joypaul V, et al. Postoperative morbidity and mortality after D1 and D2 resections for gastric cancer: preliminary results of the MRC randomised controlled surgical trial. The Surgical Cooperative Group. Lancet. 1996;347:995-9.

9. Gotoda T. Endoscopic diagnosis and treatment for early gastric cancer. Cancer Rev. 2004;2:17-37.

10. Griffith JP, Sue-Ling HM, Martin I, Dixon MF, McMahon MJ, Axon AT, et al. Preservation of the spleen improves survival after radical surgery for gastric cancer. Gut. 1995;36:684-90.

11. Hartgrink HH, van de Velde CJ, Putter H, Bonenkamp JJ, Klein Kranenbarg E, Songun I, et al. Extended lymph node dissection for gastric cancer: who may benefit? Final results of the randomized Dutch gastric cancer group trial. J Clin Oncol. 2004;22:2069-77.

12. Hokita S, Nakajo A, Uenosono Y, Sumiya TA, Kuniaki I, Natsugoe AS, et al. Characteristics and appropriate surgical treatment for gastric câncer with esophageal invasion. [0663]. In: Abstracts of the 6th International Gastric Cancer Congress, Yokohama, Japan, May 4-7, 2005.

13. Ichikura T, Ogawa T, Chochi K, Kawabata T, Sugasawa H, Mochizuki H. Minimum number of lymph nodes that should be examined for the International Union Against Cancer/American Joint Committee on Cancer TNM classification of gastric carcinoma. World J Surg. 2003;27:330-3.

14. Japanese Gastric Cancer Association. Japanese Classification of Gastric Carcinoma. 2a ed. [Comentário]. Gastric Cancer. 1998;1:10-24.

15. Katai H, Sasako M, Sano T, Fukagawa T. Gastric cancer surgery in the elderly without operative mortality. Surg Oncol. 2004;13:235-8. 
16. Kikuchi S, Katada N, Sakuramoto S, Koabayashi N, Shimao H, Sakakibara Y, et al. Factors associated with pN3 stage tumors according to the TNM classification in advanced gastric cancer. Hepatogastroenterology. 2003;50:1723-6.

17. Kitajima M. Strategies for gastric cancer treatment in the 21 st century: minimally invasive and Tailored Approaches Integrating Basic Science and clinical medicine. [PL-1]. In: Abstracts of the 6th International Gastric Cancer Congress, Yokohama, Japan, May 4-7, 2005. p.9-10.

18. Kodera Y, Schwarz RE, Nakao A. Extended lymph node dissection in gastric carcinoma: where do we stand after the Dutch and British randomized trials? J Am Coll Surg. 2002;195:855-64

19. Kunisaki C, Takahashi M, Fukushima T, Nagahori Y, Akiyama H, Makino H, et al. The influence of stage migration on the comparison of surgical outcomes between D2 gastrectomy and D3 gastrectomy (para-aortic lymph node dissection): a multi-institutional retrospective study. Am J Surg. 2008; 196:358-63.

20. Li C, Kim S, Lai JF, Oh SJ, Hyung WJ, Choi WH, et al. Lymph node dissection around the splenic artery and hilum in advanced middle third gastric carcinoma. Eur J Surg Oncol. 2008; May 1: doi:10.1016/j.ejso.2008.03.011.

21. Lim S, Muhs BE, Marcus SG, Newman E, Berman RS, Hiotis SP. Results following resection for stage IV gastric cancer; are better outcomes observed in selected patient subgroups? J Surg Oncol. 2007;95:118-22.

22. Lo SS, Wu CW, Shen KH, Hsieh MC, Lui WY. Higher morbidity and mortality after combined total gastrectomy and pancreaticosplenectomy for gastric cancer. World J Surg. 2002;26:678-82.

23. Lo SS, Wu CW, Shen KH, Hsieh MC, Lui WY. Higher morbidity and mortality after combined total gastrectomy and pancreaticosplenectomy for gastric cancer. World J Surg. 2002;26:678-82.

24. Maruyama K, Gunvén P, Okabayashi K, Sasako M, Kinoshita T. Lymph node metastases of gastric cancer. General pattern in 1931 patients. Ann Surg. 1989;210:596-602.

25. Maruyama K, Sasako M, Kinoshita T, Sano T, Katai H. Surgical treatment for gastric cancer: the Japanese approach. Semin Oncol. 1996;23:360-8.

26. Necula A, Vlad L, Iancu C, Munteanu D, Puia C, Bălă O, et al. Aspecte clinice cu implicatie prognostica in cancerul gastric--analiza a 468 cazuri de adenocarcinom gastric. Chirurgia (Bucur). 2008;103:181-8.

27. Possik RA. Fatores prognósticos de pacientes com adenocarcinoma de estômago submetidos a cirurgia radical : analise multifatorial. [Tese]. São Paulo: Faculdade de Medicina da Universidade de São Paulo; 1990.
28. Possik RA. Fatores prognósticos de pacientes com adenocarcinoma de estômago submetidos a cirurgia radical : analise multifatorial. [Tese]. São Paulo: Faculdade de Medicina da Universidade de São Paulo; 1990.

29. Rohde H, Rau E, Gebbensleben B, Stützer H, Köster R, Salzberger B, et al. What causes in-hospital mortality of surgical patients with cancer of the stomach? Scand J Gastroenterol. 1987; 22(suppl 133):76-9.

30. Sasako M, Katai H, Sano T, Maruyama K. Management of complications after gastrectomy with extended lymphadenectomy. Surg Oncol. 2000;9:31-4.

31. Sasako M. Principles of surgical treatment for curable gastric cancer. J Clin Oncol. 2003;21(Suppl):274s-275s.

32. Schwarz RE, Zagala-Nevarez K. Ethnic survival differences after gastrectomy for gastric cancer are better explained by factors specific for disease location and individual patient comorbidity. Eur J Surg Oncol. 2002;28:214-9.

33. Shiraishi N, Sato K, Yasuda K, Inomata M, Kitano S. Multivariate prognostic study on large gastric cancer. J Surg Oncol. 2007;96:14-8.

34. Siewert JR, Kestlmeier R, Busch R, Böttcher K, Roder JD, Müller J, et al. Benefits of D2 lymph node dissection for patients with gastric cancer and $\mathrm{pN} 0$ and pN1 lymph node metastases. Br J Surg. 1996;83:1144-7.

35. Sobin LH, Wittekind Ch, (Ed.). TNM : classification of malignant tumours 5a ed. New York: Wiley-Liss, 1997. 227 p.

36. Weitz J, Jaques DP, Brennan M, Karpeh M. Association of splenectomy with postoperative complications in patients with proximal gastric and gastroesophageal junction cancer. Ann Surg Oncol. 2004;11:682-9.

37. Wu CW, Hsiung CA, Lo SS, Hsieh MC, Chen JH, Li AF, et al. Stage migration influences on stage-specific survival comparison between D1 and D3 gastric cancer surgeries. Eur J Surg Oncol. 2005;31:153-7.

38. Yamamoto M, Baba H, Kakeji Y, Endo K, Ikeda Y, Toh Y, et al. Postoperative morbidity/mortality and survival rates after total gastrectomy, with splenectomy/pancreaticosplenectomy for patients with advanced gastric cancer. Hepatogastroenterology. 2004;51:298-302.

39. Yokota T, Ishiyama S, Saito T, Teshima S, Yamada Y, Iwamoto K, et al. Is tumor size a prognostic indicator for gastric carcinoma? Anticancer Res. 2002;22(6B):3673-7.

Fonte de financiamento: não hà Conflito de interesse: não há Recebido para publicação: 12/12/2009 Aceito para publicação: 17/02/2009 